\title{
VIE Regimen
}

National Cancer Institute

\section{Source}

National Cancer Institute. VIE Regimen. NCI Thesaurus. Code C9846.

A chemotherapy regimen consisting of vincristine, ifosfamide, and etoposide that may be used in the treatment of rhabdomyosarcoma. 